\title{
Black-box Models from Input-output Measurements
}

\author{
Lennart Ljung \\ Department of Electrical Engineering \\ Linköping University, SE-581 83 Linköping, Sweden \\ WWW: http://www.control.isy.liu.se \\ Email: ljung@isy.liu.se
}

October 2, 2001

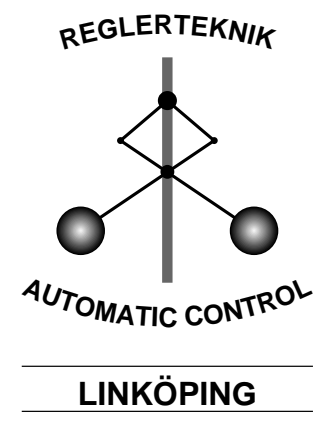

Report no.: LiTH-ISY-R-2362

For the 18th IEEE Instrumentation and Measurement Technology Conference, Budapest, 2001

Technical reports from the Automatic Control group in Linköping are available by anonymous ftp at the address ftp.control.isy.liu.se. This report is contained in the file 2362.pdf. 



\title{
Black-box Models from Input-output Measurements
}

\author{
Lennart Ljung \\ Div. of Automatic Control \\ Linköping University \\ SE-58183 Linköping, Sweden \\ email: ljung@isy.liu.se
}

Abstract - A black-box model of a system is one that does not use any particular prior knowledge of the character or physics of the relationships involved. It is therefore more a question of "curve- fitting" than "modeling". In this presentation several examples of such black-box model structures will be given. Both linear and non-linear structures are treated. Relationships between linear models, fuzzy models, neural networks and classical non-parametric models are discussed. Some reasons for the usefulness of these model types will also be given.

Ways to fit black box structures to measured input-output data are described, as well as the more fundamental (statistical) properties of the resulting models.

\section{INTRODUCTION: MODELS OF DIFFERENT COLORS}

At the heart of any estimation problem is to select a suitable model structure. A model structure is a parameterized family of candidate models of some sort, within which the search for a model is conducted.

A basic rule in estimation is not to estimate what you already know. In other words, one should utilize prior knowledge and physical insight about the system when selecting the model structure. It is customary to color-code - in shades of grey the model structure according to what type of prior knowledge has been used:

- White-box models: This is the case when a model is perfectly known; it has been possible to construct it entirely from prior knowledge and physical insight.

- Grey-box models: This is the case when some physical insight is available, but several parameters remain to be determined from observed data. It is useful to consider two sub-cases:

- Physical Modeling: A model structure can be built on physical grounds, which has a certain number of parameters to be estimated from data. This could, e.g., be a state space model of given order and structure.

- Semi-physical modeling: Physical insight is used to suggest certain nonlinear combinations of measured data signal. These new signals are then subjected to model structures of black box character.

- Black-box models: No physical insight is available or used, but the chosen model structure belongs to families that are known to have good flexibility and have been "successful in the past".

This paper deals with Black-box models for dynamical systems, for which inputs and outputs can be measured. We shall both deal with linear and non-linear systems.

First, in Section II we list the basic features of black-box modeling in terms of a simple example. In Section III we outline the basic estimation techniques used. Section IV deals with the basic trade-offs to decide the size of the structure used (essentially how many parameters are to be used: the "fineness of approximation"). So far the discussion is quite independent of the particular structure used. In Section V we turn to particular examples of linear black-box models and to issues how to choose between the possibilities, while VI similarly deals with non-linear black-box models for dynamical systems.

\section{BLACK-BOX MODELS: BASIC FEATURES}

To bring out the basic features of a black-box estimation problem, let us study a simple example. Suppose the problem is to estimate an unknown function $g_{0}(x),-1 \leq x \leq 1$. The observations we have are noise measurements $y(k)$ at points $x_{k}$ which we may or may not choose ourselves:

$$
y(k)=g_{0}\left(x_{k}\right)+e(k)
$$

How to approach this problem? One way or another we must decide "where to look for" $g$. We could, e.g., have the information that $g$ is a third order polynomial. This would lead to the - in this case - grey box model structure

$$
g(x, \theta)=\theta_{1}+\theta_{2} x+\theta_{3} x^{2}+\ldots+\theta_{n} x^{n-1}
$$

with $n=4$, and we would estimate the parameter vector $\theta$ from the observations $y$, using e.g. the classical least squares method.

Now suppose that we have no structural information at all about $g 8 /$ b $\$$ Mud it, e.g. it is an analytical function, or that it is piecewise constant or something like that. In this situation, we could still use (2), but now as black-box model: if we assume $g$ to be analytic we know that it can be approximated arbitrarily well by a 
polynomial. The necessary order $n$ would not be known, and we would have to find a good value of it using some suitable scheme.

Note that there are several alternatives in this black-box situation: We could use rational approximations:

$$
g(x, \theta)=\frac{\theta_{1}+\theta_{2} x+\theta_{3} x^{2}+\ldots+\theta_{n} x^{n-1}}{1+\theta_{n+1} x+\theta_{n+2} x^{2}+\ldots+\theta_{n+m-1} x^{m-1}}
$$

or Fourier series expansions

$$
g(x, \theta)=\theta_{0}+\sum_{\ell=1}^{n} \theta_{2 \ell-1} \cos (\ell \pi x)+\theta_{2 \ell} \sin (\ell \pi x)
$$

Alternatively, we could approximate the function by piecewise constant functions, as illustrated in Figure 1. We shall

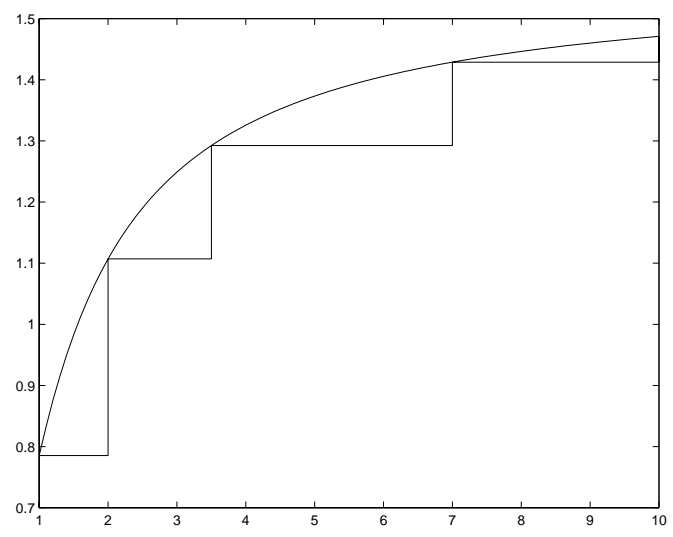

Fig. 1. A piece-wise constant approximation.

in Section VI return to a formal model description of the parameterization in this figure.

It is now clear that the basic steps of black-box modeling are as follows:

1. Choose the "type" of model structure class. (For example, in the case above, Fourier transform, rational function, or piecewise constant.)

2. Determine the "size" of this model (i.e. the number of parameters, $n$ ). This will correspond to how "fine" the approximation is.

3. Use observed data both to estimate the numerical values of the parameters and to select a suitable value of $n$.

We shall generally denote a model structure for the observations by

$$
\hat{y}(k \mid \theta)=g\left(x_{k}, \theta\right)
$$

It is to interpreted so that $\hat{y}(k \mid \theta)$ is the predicted value of the observation $y(k)$ assuming the function can be described by the parameter vector $\theta$.
We shall also generally denote all measurements available up to time $N$ by

$$
Z^{N}
$$

\section{ESTIMATION TECHNIQUES AND BASIC PROPERTIES}

In this section we shall deal with issues that are independent of model structure. Principles and algorithms for fitting models to data, as well as the general properties of the estimated models are all model-structure independent and equally well applicable to, say, linear ARMAX models and Neural Network models, to be discussed later in this paper.

\section{A. Criterion of Fit}

It suggests itself that the basic least-squares like approach is a natural approach, even when the predictor $\hat{y}(t \mid \theta)$ is a more general function of $\theta$ :

$$
\hat{\theta}_{N}=\arg \min _{\theta} V_{N}\left(\theta, Z^{N}\right)
$$

where

$$
V_{N}\left(\theta, Z^{N}\right)=\frac{1}{N} \sum_{t=1}^{N}\|y(t)-\hat{y}(t \mid \theta)\|^{2}
$$

We shall also use the following notation for the discrepancy between measurement and predicted value

$$
\varepsilon(t, \theta)=y(t)-\hat{y}(t, \mid \theta)
$$

This procedure is natural and pragmatic - we can think of it as "curve-fitting" between $y(t)$ and $\hat{y}(t \mid \theta)$. It also has several statistical and information theoretic interpretations. Most importantly, if the noise source in the system is supposed to be a Gaussian sequence of independent random variables $\{e(t)\}$ then (7) becomes the Maximum Likelihood estimate (MLE).

If $\hat{y}(k \mid \theta)$ is a linear function of $\theta$ the minimization problem (7) is easily solved. In more general cases the minimization will have to be carried out by iterative (local) search for the minimum:

$$
\hat{\theta}_{N}^{(i+1)}=\hat{\theta}_{N}^{(i)}+\mu f\left(Z^{N}, \hat{\theta}_{N}^{(i)}\right)
$$

where $f$ typically is related to the gradient of $V_{N}$, like the Gauss-Newton direction. See, e.g. [1], Chapter 10.

It is also quite useful work with a modified criterion

$$
W_{N}\left(\theta, Z^{N}\right)=V_{N}\left(Z^{N}\right)+\delta\|\theta\|^{2}
$$

with $V_{N}$ defined by (8). This is known as regularization. It may be noted that stopping the iterations $(i)$ in (10) before the minimum has been reached has the same effect as regularization. See, e.g., [2]. 


\section{B. Convergence as $N \rightarrow \infty$}

An essential question is, of course, what will be the properties of the estimate resulting from (8). These will naturally depend on the properties of the data record $Z^{N}$. It is in general a difficult problem to characterize the quality of $\hat{\theta}_{N}$ exactly. One normally has to be content with the asymptotic properties of $\hat{\theta}_{N}$ as the number of data, $N$, tends to infinity.

It is an important aspect of the general identification method (8) that the asymptotic properties of the resulting estimate can be expressed in general terms for arbitrary model parameterizations.

The first basic result is the following one:

$$
\begin{gathered}
\hat{\theta}_{N} \rightarrow \theta^{*} \text { as } N \rightarrow \infty \text { where } \\
\theta^{*}=\arg \min _{\theta} E\|\varepsilon(t, \theta)\|^{2}
\end{gathered}
$$

That is, as more and more data become available, the estimate converges to that value $\theta^{*}$, that would minimize the expected value of the "norm" of the prediction errors. This is in a sense the best possible approximation of the true system that is available within the model structure. The expectation $E$ in (13) is taken with respect to all random disturbances that affect the data and it also includes averaging over the' "input properties" (in the example of Section II, this would be the distribution of the values of $x_{k}$.). This means, in particular, that $\theta^{*}$ will make $\hat{y}\left(t \mid \theta^{*}\right)$ a good approximation of $y(t)$ with respect to those aspects of the system that are enhanced by the conditions at hand, when the data were collected.

\section{Asymptotic Distribution}

Once the convergence issue has been settled, the next question is how fast the limit is approached. This is dealt with by considering the asymptotic distribution of the estimate. The basic result is the following one: If $\left\{\varepsilon\left(t, \theta^{*}\right)\right\}$ is approximately white noise, then the random vector $\sqrt{N}\left(\hat{\theta}_{N}-\theta^{*}\right)$ converges in distribution to the normal distribution with zero mean and the covariance matrix of $\hat{\theta}_{N}$ is approximately given by

$$
P_{\theta}=\lambda\left[E \psi(t) \psi^{T}(t)\right]^{-1}
$$

where

$$
\begin{aligned}
\lambda & =E \varepsilon^{2}\left(t, \theta^{*}\right) \\
\psi(t) & =\left.\frac{d}{d \theta} \hat{y}(t \mid \theta)\right|_{\theta=\theta^{*}}
\end{aligned}
$$

This means that the convergence rate of $\hat{\theta}_{N}$ towards $\theta^{*}$ is $1 / \sqrt{N}$. Think of $\psi$ as the sensitivity derivative of the predictor with respect to the parameters. It is also used in the actual numerical search algorithm (10). Then (14) says that the covariance matrix for $\hat{\theta}_{N}$ is proportional to the inverse of the covariance matrix of this sensitivity derivative. This is a quite natural result.

The result (14) - (15) is general and holds for all model structures, both linear and non-linear ones, subject only to some regularity and smoothness conditions. They are also fairly natural, and will give the guidelines for all user choices involved in the process of identification. Of particular importance is that the asymptotic covariance matrix (14) equals the Cramér-Rao lower bound, if the disturbances are Gaussian. That is to say, prediction error methods give the optimal asymptotic properties. See [1] for more details around this.

\section{CHOICE OF “TYPE” AND “SIZE” OF MODEL}

\section{A. Bias-Variance Trade-off}

The obtained model $g\left(x, \hat{\theta}_{N}\right)$ will be in error in two ways:

1. First, there will be a discrepancy between the limit model $g\left(x, \theta^{*}\right)$ and the true function $g_{0}(x)$, since our structure assumptions are not correct, e.g. the function is not piecewise constant. This error we call a bias error, or a modelmismatch error.

2. Second, there will be a discrepancy between the actual estimate $\hat{\theta}_{N}$ and the limit value. This is due to the noisecorrupted measurements (the term $e(k)$ in (1)). This error will be called a variance error, and can be measured by the covariance matrix (14).

It should be clear that there is a trade-off between these aspects: To get a smaller bias error, we should, e.g., use a finer grid for the piece-wise approximation. This in turn would mean that fewer observed data can be used to estimate each level of the piece-wise function approximation, which leads to large variance for these estimates.

To deal with the bias-variance trade-off is thus at the heart of the black-box model structure selection.

Some quite general expressions for the expected model fit, that are independent of the model structure, can be developed, and these will be dealt with in this section.

\section{B. An Expression for the Expected Mean-Square Error}

Let us measure the (average) fit between any model (5) and the true system as

$$
\bar{V}(\theta)=E|y(t)-\hat{y}(t \mid \theta)|^{2}
$$

Here expectation $E$ is over the data properties (i.e. expectation over " $Z$ " with the notation (6)).

Before we continue, let us note the very important aspect that the fit $\bar{V}$ will depend, not only on the model and the true system, but also on data properties. In the simple case of Section II this would be the distribution of the observation points 
$x_{k}, k=1,2, \ldots$ In the case of dynamical systems it involves input spectra, possible feedback, etc. We shall say that the fit depends on the experimental conditions.

The estimated model parameter $\hat{\theta}_{N}$ is a random variable, because it is constructed from observed data, that can be described as random variables. To evaluate the model fit, we then take the expectation of $\bar{V}\left(\hat{\theta}_{N}\right)$ with respect to the estimation data. That gives our measure

$$
F_{N}=E \bar{V}\left(\hat{\theta}_{N}\right)
$$

The rather remarkable fact is that if $F_{N}$ is evaluated for data with the same properties as those of the estimation data, then, asymptotically in $N$, (see, e.g., [1], Chapter 16)

$$
F_{N} \approx \bar{V}\left(\theta^{*}\right)\left(1+\frac{\operatorname{dim} \theta}{N}\right)
$$

Here $\theta^{*}$ is the value that minimizes the expected value of the criterion (8). The notation $\operatorname{dim} \theta$ means the number of estimated parameters. The result also assumes that the model structure is successful in the sense that $\varepsilon(t)$ is approximately white noise.

It is quite important to note that the number $\operatorname{dim} \theta$ in (18) will be changed to the number of eigenvalues of $\bar{V}^{\prime \prime}(\theta)$ (the Hessian of $\bar{V}$ ) that are larger than $\delta$ in case the regularized loss function (11) is minimized to determine the estimate. We can think of this number as the efficient number of parameters. In a sense, we are "offering" more parameters in the structure, than are actually "used" by the data in the resulting model.

Despite the reservations about the formal validity of (18), it carries a most important conceptual message: If a model is evaluated on a data set with the same properties as the estimation data, then the fit will not depend on the data properties, and it will depend on the model structure only in terms of the number of parameters used and of the best fit offered within the structure.

The expression (18) clearly shows the trade off between variance and bias. The more parameters used by the structure (corresponding to a higher dimension of $\theta$ and/or a lower value of the regularization parameter $\delta$ ) the higher the variance term, but at the same the lower the fit $\bar{V}\left(\theta^{*}\right)$. The trade off is thus to increase the efficient number of parameters only to that point that the improvement of fit per parameter exceeds $\bar{V}\left(\theta^{*}\right) / N$. This can be achieved by estimating $F_{N}$ in (17) by evaluating the loss function at $\hat{\theta}_{N}$ for a validation data set. It can also be achieved by Akaike (or Akaike-like) procedures, [3], balancing the variance term in (18) against the fit improvement.

The expression can be rewritten as follows. Let $g_{0}(t)$ denote the "true" one step ahead prediction of $y(t)$, and let

$$
W(\theta)=E\left|g_{0}(t)-\hat{y}(t \mid \theta)\right|^{2}
$$

and let

$$
\left.\lambda=E \mid y(t)-g_{0}(t)\right)\left.\right|^{2}
$$

In Section II this is just the variance of $e(t)$. In the more general cases of dynamic models to be considered later, $\lambda$ is the innovations variance, i.e., that part of $y(t)$ that cannot be predicted from the past. Moreover $W\left(\theta^{*}\right)$ is the bias error, i.e. the discrepancy between the true predictor and the best one available in the model structure. Under the same assumptions as above, (18) can be rewritten as

$$
F_{N} \approx \lambda+W\left(\theta^{*}\right)+\lambda \frac{\operatorname{dim} \theta}{N}
$$

The three terms constituting the model error then have the following interpretations

- $\lambda$ is the unavoidable error, stemming from the fact that the output cannot be exactly predicted, even with perfect system knowledge.

- $W\left(\theta^{*}\right)$ is the bias error. It depends on the model structure, and on the experimental conditions. It will typically decrease as $\operatorname{dim} \theta$ increases.

- The last term is the variance error. It is proportional to the (efficient) number of estimated parameters and inversely proportional to the number of data points. It does not depend on the particular model structure or the experimental conditions.

\section{A Procedure in Practice}

A pragmatic and quite useful way to strike a good balance between variance and bias, i.e., to minimize (21) w.r.t. $\operatorname{dim} \theta$ is as follows:

- Split the observed data into an estimation set and a validation set

- Estimate models, using the estimation data set, for a number of different sizes of the parameter vector.

- Compute the value of the criterion for each of these models, using the validation data.

- Pick as your final model the one that minimizes the fit for validation data.

An alternative is to offer a large number of parameters in a fixed model structure and use the regularized criterion (11). Estimate several models, as you turn the "knob" $\delta$ from larger values towards zero and evaluate the obtained models as above.

\section{LINEAR BLACK-BOX MODELS}

\section{A. Linear Models and Estimating Frequency Functions}

A linear system is uniquely defined and described by its frequency function $G\left(e^{i \omega}\right)$ (i.e. the Fourier transform of its impulse response. We could therefore link estimation of linear systems directly to the function estimation problem (1), taking 
$x_{k}=e^{i \omega_{k}}$ and allowing $g$ to be complex-valued. With observations of the input-output being directly taken from, or transformed to the frequency domain ( $y$ would here be uncertain observations of the frequency response at certain frequencies) we have a straightforward function estimation problem.

One question is then how to parameterize $G\left(e^{i \omega}\right)$. It could be done by piece-wise constant functions, which is closely related to standard spectral analysis (see Problem 7G.2 in [1]). See also e.g. [4]. Otherwise it is more common to parameterize them as rational functions as in (3). Direct fits of rational frequency functions to observed frequency domain data is treated comprehensively in [5], and in, e.g. Section 7.7 of [1].

\section{B. Time-domain Data and General Linear Models}

If the observations $y$ to be used for the model fit are inputoutput data in the time domain, we proceed as follows: Assume that the data have been generated according to

$$
y(t)=G(q, \theta) u(t)+H(q, \theta) e(t)
$$

where $e$ is white noise (unpredictable), $q$ is the forward shift operator and $H$ is monic (that is, its expansion in $q^{-1}$ starts with the identity matrix). We also assume that $G$ contains a delay. Rewrite (22) as

$$
y(t)=\left[I-H^{-1}(q, \theta)\right] y(t)+H^{-1}(q, \theta) G(q, \theta) u(t)+e(t)
$$

The first term in the RHS only contains $y(t-k), k \leq 1$ so the natural predictor of $y(t)$, based on past data will be given by

$$
\begin{aligned}
\hat{y}(t \mid \theta) & =W_{y}(q, \theta) y(t)+W_{u}(q, \theta) u(t) \\
W_{y}(q, \theta) & =\left[I-H^{-1}(q, \theta)\right], \\
W_{u}(q, \theta) & =H^{-1}(q, \theta) G(q, \theta)
\end{aligned}
$$

It will be required that $\theta$ are constrained to values such that the filters $H^{-1} G$ and $H^{-1}$ are stable.

\section{Linear Input-output Black-box models}

In the black-box case, a very natural approach is to describe $G$ and $H$ in (22) as rational transfer functions in the shift (delay) operator with unknown numerator and denominator polynomial asn in (3). We would then have

$$
\begin{aligned}
G(q, \theta) & =\frac{B(q)}{F(q)} \\
& =\frac{b_{1} q^{-n k}+b_{2} q^{-n k-1}+\cdots+b_{n b} q^{-n k-n b+1}}{1+f_{1} q^{-1}+\cdots+f_{n f} q^{-n f}}
\end{aligned}
$$

Then

$$
\eta(t)=G(q, \theta) u(t)
$$

is a shorthand notation for the relationship

$$
\begin{aligned}
\eta(t) & +f_{1} \eta(t-1)+\cdots+f_{n f} \eta(t-n f) \\
& =b_{1} u(t-n k)+\cdots+b_{n b} u(t-(n b+n k-1))
\end{aligned}
$$

Here, there is a time delay of $n k$ samples.

In the same way the disturbance transfer function can be written

$$
H(q, \theta)=\frac{C(q)}{D(q)}=\frac{1+c_{1} q^{-1}+\cdots+c_{n c} q^{-n c}}{1+d_{1} q^{-1}+\cdots+d_{n d} q^{-n d}}
$$

The parameter vector $\theta$ thus contains the coefficients $b_{i}, c_{i}, d_{i}$, and $f_{i}$ of the transfer functions. This model is thus described by five structural parameters: $n b, n c, n d, n f$, and $n k$ and is known as the Box-Jenkins (BJ) model.

An important special case is when the properties of the disturbance signals are not modeled, and the noise model $H(q)$ is chosen to be $H(q) \equiv 1$; that is, $n c=n d=0$. This special case is known as an output error $(O E)$ model since the noise source $e(t)=v(t)$ will then be the difference (error) between the actual output and the noise-free output.

A common variant is to use the same denominator for $G$ and $H$ :

$$
F(q)=D(q)=A(q)=1+a_{1} q^{-1}+\cdots+a_{n a} q^{-n a}
$$

Multiplying both sides of (26)-(29) by $A(q)$ then gives

$$
A(q) y(t)=B(q) u(t)+C(q) e(t)
$$

This model is known as the ARMAX model. The name is derived from the fact that $A(q) y(t)$ represents an AutoRegression and $C(q) e(t)$ a Moving Average of white noise, while $B(q) u(t)$ represents an eXtra input (or with econometric terminology, an eXogenous variable).

The special case $C(q)=1$ gives the much used $A R X$ model. In Figure 2 the different models are depicted.

\section{Linear State-space Black-box models}

An important use of state-space models is to incorporate any available physical insights into the system. It can however also be used for black-box modeling, both in continuous and discrete time. A discrete time innovations form is

$$
\begin{aligned}
x(t+1) & =A(\theta) x(t)+B(\theta) u(t)+K(\theta) e(t) \\
y(t) & =C(\theta) x(t)+e(t)
\end{aligned}
$$

If the parameterization is done so that (32) covers all linear system of a certain order as the parameter $\theta$ ranges of a certain set, then this will give a black-box model. For example, if $\theta$ parameterizes every matrix element in an independent way, this will clearly correspond to a black box model. We shall 


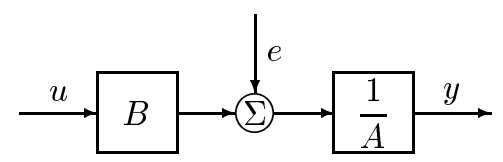

ARX

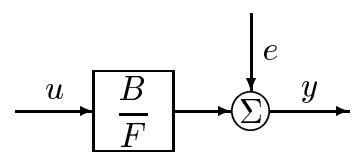

OE

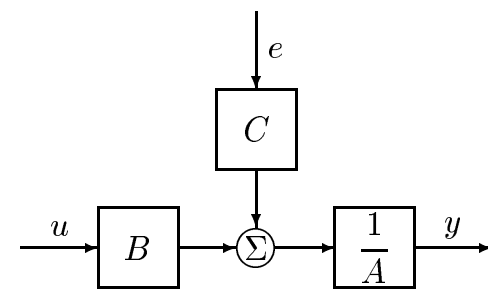

ARMAX

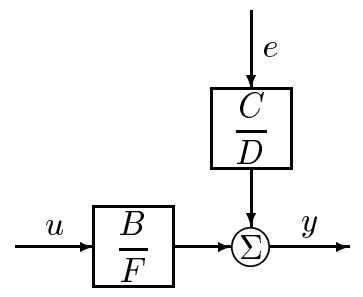

Fig. 2. The different linear black-box models

call that a full parameterization. The only structure parameter to determine is then the model order, $\operatorname{dim} x$. While this may be wasteful in the sense that $\operatorname{dim} \theta$ will be much larger than necessary, it gives the important advantage that is also covers all choices of coordinate basis in the state space and thus includes those parameterizations that are best conditioned.

(A canonical parameterization only needs $\operatorname{dim} \theta=n(2 p+m)$ parameters with $n=\operatorname{dim} x$ and $p$ and $m$ being the number of inputs and outputs. On the other hand, some overlapping such parameterizations will be necessary to cover all $n$ :th order systems if $p>1$, and they will typically not be very well conditioned. See e.g. Appendix 4A in [1].)

An advantage with a full parameterization of (32) is that so called subspace methods (see e.g. [6]) allow efficient noniterative estimation of all the matrices involved. The model can then be further refined by minimization over $\theta$ in the criterion (8). This can still be done efficiently, even if the parameterization is full, see [7].

\section{E. Some Practical Aspects}

To choose the order(s) of the linear black box models one essentially follows the procedure described at the end of Section IV. That is, a number of different orders are tried out, and the resulting models' ability to reproduce validation data is inspected. For ARX models, this can be done very efficiently over many models simultaneously.

The choice between the different members of the black-box families in Figure 2 can be guided by the following comments:

- Models that have a common denominator for $G$ and $H$, that is ARMAX and ARX are suitable when the dominating disturbances are load disturbances, that enter through the same dynamics as the input.

- The BJ model is suitable when there is no natural connection between the systems dynamics and the disturbances (like measurement noise).

- The OE model has the advantage that a consistent estimate of $G$ can be obtained even if the disturbance properties are not correctly modeled (provided the system operates in open loop).

\section{NON-LINEAR BLACK-BOX MODELS}

There is clearly a wide variety of non-linear models. One possibility that allows inclusion of detailed physical prior information is to build non-linear state space models, analogous to (32). Another possibility of the grey-box modeling type is to use semi-physical modeling mentioned in the introduction. This section however deals with black-box models. We follow the presentation in Chapter 5 of [1], to which we refer for any details.

\section{A. Non-linear Black-box Models}

We shall generally let $\varphi$ contain a finite number of past input output data:

$$
\varphi(t)=[y(t-1), \ldots, y(t-n), u(t-k), \ldots, u(t-k-m)]^{T}
$$

and the predictor for $y(t)$ is allowed to to be a general function of $\varphi(t)$ :

$$
\hat{y}(t)=g(\varphi(t))
$$

We are thus, again, back at the simple example (1). The mapping $g$ can be parameterized as a function expansion

$$
g(\varphi, \theta)=\sum_{k=1}^{n} \alpha_{k} \kappa\left(\beta_{k}\left(\varphi-\gamma_{k}\right)\right)
$$

Here, $\kappa$ is a "mother basis function", from which the actual functions in the function expansion are created by dilation (parameter $\beta$ ) and translation (parameter $\gamma$ ). For example, with $\kappa=\cos$ we would get Fourier series expansion with $\beta$ as frequency and $\gamma$ as phase. More common are cases where $\kappa$ is a unit pulse. With that choice, (36) can describe any piecewise constant function, where the granularity of the approximation is governed by the dilation parameter $\beta$. Compared to Figure 1 we would in that case have 
- $n=4$,

- $\gamma_{1}=1, \gamma_{2}=2, \gamma_{3}=3.5, \gamma_{4}=7$,

- $\beta_{1}=1, \beta_{2}=2 / 3, \beta_{3}=1 / 3.5, \beta_{4}=1 / 3$

- $\alpha_{1}=0.79, \alpha_{2}=1.1, \alpha_{3}=1.3, \alpha_{4}=1.43$

A related choice is a soft version of a unit pulse, such as the Gaussian bell. Alternatively, $\kappa$ could be a unit step (which also gives piecewise constant functions), or a soft step, such as the sigmoid.

Typically $\kappa$ is in all cases a function of a scalar variable. When $\varphi$ is a column vector, the interpretation of the argument of $\kappa$ can be made in different ways:

- If $\beta$ is a row vector $\beta(\varphi-\gamma)$ is a scalar, so the term in question is constant along a hyperplane. This is called the ridge approach, and is typical for sigmoidal neural networks.

- Interpreting the argument as $\|\varphi-\gamma\|_{\beta}$ as a quadratic norm with the positive semidefinite matrix $\beta$ as a quadratic form, gives terms that are constant on spheres (in the $\beta$ norm) around $\gamma$. This is called the radial approach. Radial basis neural networks are common examples of this.

- Letting $\kappa$ be interpreted as the product of $\kappa$-functions applied to each of the components of $\varphi$, gives yet another approach, known as the tensor approach. The functions used in (neuro-)fuzzy modeling are typical examples of this.

Note that the model structure is entirely determined by:

- The scalar valued function $\kappa(x)$ of a scalar variable $x$.

- The way the basis functions are expanded to depend on a vector $\varphi$.

The parameterization in terms of $\theta$ can be characterized by three types of parameters:

- The coordinates $\alpha$

- The scale or dilation parameters $\beta$

- The location parameters $\gamma$

These three parameter types affect the model in quite different ways. The coordinates enter linearly, which means that (36) is a linear regression for fixed scale and location parameters.

\section{B. Approximation Issues}

A key issue is how well the function expansion is capable of approximating any possible "true system" $g_{0}(\varphi)$. There is rather extensive literature on this subject. See, e.g., [8]. for an identification oriented survey,

The bottom line can be expressed as follows For almost any choice of $\kappa(x)$ - except being a polynomial - the expansion (36) can approximate any "reasonable" function $g_{0}(\varphi)$ arbitrarily well for sufficiently large $n$.
It is not difficult to understand this. It is sufficient to check that the delta function - or the indicator function for arbitrarily small areas - can be arbitrarily well approximated within the expansion. Then clearly all reasonable functions can also be approximated. For a a simple unit pulse function $\kappa$, with radial construction this is immediate: It is itself a delta function approximator.

The question of how efficient the expansion is, i.e., how large $n$ is required to achieve a certain degree of approximation, is more difficult, and has no general answer. See, e.g. [9]. We may point to the following aspects:

- If the scale and location parameters $\beta$ and $\gamma$ are allowed to depend on the function $g_{0}$ to be approximated, then the number of terms $n$ required for a certain degree of approximation is much less than if $\beta_{k}, \gamma_{k}, k=1, \ldots$ is an a priori fixed sequence. To realize this, consider the following simple example:

\section{EXAMPle VI.1: Piece-wise Constant Functions}

(From [1]) Suppose we use, as in Figure 1 piece-wise constant functions to approximate any scalar valued function of a scalar variable $\varphi$ :

$$
\begin{aligned}
& g_{0}(\varphi), \quad 0 \leq \varphi \leq B \\
& \hat{g}_{n}(\varphi)=\sum_{k=1}^{n} \alpha_{k} \kappa\left(\beta_{k}\left(\varphi-\gamma_{k}\right)\right)
\end{aligned}
$$

where $\kappa$ is the unit pulse. Suppose that we require $\sup _{\varphi} \mid g_{0}(\varphi)-$ $\hat{g}_{n}(\varphi) \mid \leq \epsilon$ and we know a bound on the derivative of $g_{0}: \sup \left|g_{0}^{\prime}(\varphi)\right| \leq$ $C$. For (38) to be able to deliver such a good approximation for any such function $g_{0}$ we need to take $\gamma_{k}=k \Delta, \beta_{k}=1 / \Delta$ with $\Delta \leq 2 \epsilon / C$, i.e., we need $n \geq C B /(2 \epsilon)$. That is, we need a fine grid $\Delta$ that is prepared for the worst case $\left|g_{0}^{\prime}(\varphi)\right|=C$ at any point.

If the actual function to be approximated turns out to be much smoother, and has a large derivative only in a small interval, we can adapt the choice of $\beta_{k}=1 / \Delta_{k}$ so that $\Delta_{k} \approx 2 \epsilon /\left|g_{0}^{\prime}\left(\varphi^{*}\right)\right|$ for the interval around $\gamma_{k}=k \Delta_{k} \approx \varphi^{*}$ which may give the desired degree of approximation with much fewer terms in the expansion.

- For the local, radial approach the number of terms required to achieve a certain degree of approximation $\delta$ of a $p$ times differentiable function in $\mathcal{R}^{d}(\operatorname{dim} \varphi=d)$ is proportional to

$$
n \sim \frac{1}{\delta^{(d / p)}}, \quad \delta \ll 1
$$

It thus increases exponentially with the number of regressors. This is often referred to as the curse of dimensionality.

\section{Neural Networks}

Neural networks have become a very popular choice of model structure in recent years. The name refers to certain structural similarities with the neural synapse system in animals. From our perspective these models correspond to certain choices in the general function expansion. 


\section{Sigmoid Neural Networks.}

The combination of the model expansion (36), with a ridge interpretation of $\beta(\varphi-\gamma)$ and the sigmoid choice $\kappa(x)=$ $1 /\left(1+e^{x}\right)$ for mother function, gives the celebrated one hidden layer feedforward sigmoid neural net.

Wavelet and Radial Basis Networks.

The combination of the Gaussian bell type mother function and the radial interpretation of $\beta(\varphi-\gamma)$ is found in both wavelet networks and radial basis neural networks.

\section{Wavelets}

Wavelet decomposition is a typical example for the use of local basis functions. Loosely speaking, the "mother basis function" is dilated and translated to form a wavelet basis. In this context it is common to let the expansion (36) be doubly indexed according to scale and location, and use the specific choices (for one dimensional case) $\beta_{j}=2^{j}$ and $\gamma_{k}=k$. This gives, in our notation,

$$
g_{j, k}(\varphi)=2^{j / 2} \kappa\left(2^{j} \varphi-k\right) j, k \in \mathbf{Z} .
$$

Note the multi-resolution capabilities, i.e. several different scale parameters are used simultaneously, so that the intervals are multiply covered using basis functions of different resolutions (i.e. different scale parameters).

\section{E. Nonparametric Regression in Statistics}

Estimation of an unstructured, unknown function as in (1) is a much studied problem in statistics. See among many references, e.g. [10] and [11].

Kernel Estimators.

A well known example for use of local basis functions is Kernel estimators. A kernel function $\kappa(\cdot)$ is typically a bell-shaped function, and the kernel estimator has the form

$$
g(\varphi)=\sum_{k=1}^{n} \alpha_{k} \kappa\left(\left\|\frac{\varphi-\gamma_{k}}{h}\right\|\right)
$$

where $h$ is a small positive number, $\gamma_{k}$ are given points in the space of regression vector $\varphi$. This clearly is a special case of (36) with one fixed scale parameter $h=1 / \beta$ for all the basis functions. This scale parameter is typically tuned to the problem, though. A common choice of $\kappa$ in this case is the Epanechnikov kernel:

$$
\kappa(x)= \begin{cases}1-x^{2} & \text { for }|x|<1 \\ 0 & \text { for }|x| \geq 1\end{cases}
$$

Nearest Neighbors or Interpolation

The nearest neighbor approach to identification has a strong intuitive appeal: When encountered with a new value $\varphi^{*}$ we look into the past data $Z^{N}$ to find that regression vector $\varphi(t)=$ $\varphi^{\dagger}$ that is closest to $\varphi^{*}$. We then associate the regressor $\varphi^{*}$ with the measurement $y(t)$ corresponding to $\varphi(t)=\varphi^{\dagger}$. This is achieved in (36) by picking scale and location parameters so that each term "contains" exactly one observation.

B-Splines.

B-splines are local basis functions which are piece-wise polynomials. The connections of the pieces of polynomials have continuous derivatives up to a certain order, depending on the degree of the polynomials. Splines are very nice functions, since they are computationally very simple and can be made as smooth as desired. For these reasons, they have been widely used in classic interpolation problems.

\section{F. Fuzzy Models}

Fuzzy models are essentially an attempt to incorporate some physical insight of non-mathematical nature into an otherwise black-box model. They are based on verbal and imprecise descriptions on the relationships between the measured signals in a system. The fuzzy models typically consist of so-called rule bases, but can be cast exactly into the framework of model structures of the class (36). In this case, the basis functions are constructed from the fuzzy set membership functions and inference rules of combining fuzzy rules and how to "defuzzify" the results. When the fuzzy models contain parameters to be adjusted, they are also called neuro-fuzzy models.

We refer to Section 5.6 in [1] for the details of this. The bottom line is that the terms of (36) are obtained from the parameterized membership functions as

$$
g_{k}(\varphi, \beta, \gamma)=\prod_{j=1}^{d} \kappa_{j}\left(\beta_{k}^{j}\left(\varphi_{j}-\gamma_{k}^{j}\right)\right)
$$

Here $\kappa_{j}$ would be a basic membership function (often piecewise linear) for the $j: t h$ component of $\varphi$, describing, e.g. the temperature. Moreover $\beta$ would describe how quickly the transition from say cold to hot would take place, while $\gamma$ would give the temperature around which this transition takes place. Finally $\alpha$ in (36) would the the typical expected output for the particular combination of regressor values given by (43).

We are thus back to the basic situation of (36) and where the expansion into the $d$-dimensional regressor space is obtained by the tensor product construction.

Normally, not all of the parameters $\alpha_{k}, \beta_{k}$ and $\gamma_{k}$ should be freely adjustable. If the fuzzy partition is fixed and not adjustable (i.e $\beta$ and $\gamma$ fixed), then we get a particular case of the kernel estimate (41), which is also a linear regression model. 
Thus fuzzy models are just particular instances of the general model structure (36).

One of the major potential advantages is that the fuzzy rule basis may give functions $g_{j}$ that reflect (verbal) physical insight about the system. This may be useful also to come up with reasonable initial values of the dilation and location parameters to be estimated. One should realize, though, that the knowledge encoded in a fuzzy rule base may be nullified if many parameters are let loose.

\section{CONCLUSIONS}

We have illustrated the kinship between many model structures used to identify dynamical systems. We have treated the case there is no particular physical knowledge about the systems properties: The black-box case. The archetypical problem of estimation an unknown function from noisy observations of its values serves as a good guide both for linear and non-linear dynamical system.

A key issue is to find a flexible enough model parameterization. There are many such parameterizations available, known under a variety of names. It is not possible to point to any particular one that would be uniformly best. Experience and available software dictates the choice in practice.

Another key issue is to find a suitable "size" or "fineness of approximation" of the model structure. The basic advice in this respect is to estimate models of different complexity and evaluate them using validation data. A good way of constraining the flexibility of certain model classes is to use a regularized criterion of fit.

\section{References}

[1] L. Ljung, System Identification - Theory for the User, Prentice-Hall, Upper Saddle River, N.J., 2nd edition, 1999.

[2] J. Sjöberg, Q. Zhang, L. Ljung, A. Benveniste, B. Delyon, P.Y. Glorennec, H. Hjalmarsson, and A. Juditsky, "Nonlinear black-box modeling in system identification: A unified overview," Automatica, vol. 31, no. 12, pp. 1691-1724, 1995.

[3] H. Akaike, "A new look at the statistical model identification," IEEE Transactions on Automatic Control, vol. AC-19, pp. 716-723, 1974.

[4] L.P. Wang and W. R. Cluett, "Frequency-sampling filters: An imporves model structure for step-response identification," Automatica, vol. 33, no. 5, pp. 939-944, May 1997.

[5] J. Schoukens and R. Pintelon, Identification of Linear Systems: A Practical Guideline to Accurate Modeling, Pergamon Press, London (U.K.), 1991.

[6] P. Van Overschee and B. DeMoor, Subspace Identification of Linear Systems: Theory, Implementation, Applications, Kluwer Academic Publishers, 1996.

[7] T. McKelvey and A. Helmersson, "State-space parametrizations of multivariable linear systems using tridiagonal matrix forms," in IEEE Proceedings of the 35th Conference on Decision and Control, Kobe, Japan, December 1996, pp. 3654-3659.

[8] A. Juditsky, H. Hjalmarsson, A. Benveniste, B. Delyon, L. Ljung, J. Sjöberg, and Q. Zhang, "Nonlinear black-box modeling in system identification: Mathematical foundations," Automatica, vol. 31, no. 12, pp. 1724-1750, 1995.

[9] A. Barron, "Universal approximation bounds for superpositions of a sigmoidal function," IEEE Trans. Inf. Theory, vol. IT-39, pp. 930-945, 1993.
[10] C.J. Stone, "Consistent non-parametric regression (with discussion)," Ann. Statist., vol. 5, pp. 595-645, 1977.

[11] M.P. Wand and M.C. Jones, Kernel Smoothing, Number 60 in Monographs on Statistics and Applied Probability. Chapman \& Hall, 1995. 